\title{
Survey and Analysis of Metro Passenger's Dynamic Guidance Information Acceptability
}

\author{
Wang Zhiqiang ${ }^{1}$, Huang Yage ${ }^{1}$ \\ ${ }^{1}$ Urban Rail Transit Institute, Soochow University, Suzhou 215006, China; PH15862513767; E-Mail: \\ wangzhiqiang1980@163.com
}

Key Words: metro passenger; dynamic guidance information; acceptability; questionnaire survey Abstract. When metro system encounter unforeseen circumstances leads to the system operating abnormally, passengers travel plan's adjustments need to rely on dynamic guidance information to complete, therefore, metro dynamic guidance information's completion degree have significant influence to network passengers' guidance and evacuation under abnormal circumstance. This paper use questionnaire survey method, collect metro passengers evaluation data to common dynamic guidance information, based on the survey result, analyze the availability, clarity and comprehensibility characteristics of dynamic guidance information in various medium, find strengths and weaknesses of various types dynamic information, puts forward its application condition and improvement direction, and combine with the passengers' individual characteristics data, find out the passenger's attributes which influence the information's acceptability. Understand passenger's acceptance preferences, can make dynamic guidance information release plan aim at the passengers' component characteristics, get passengers' organization and evacuation achieve better results, so that promote the passenger service level and strengthen metro system's emergency handling ability.

\section{Preface}

The metro passenger guidance system include static guidance signs and dynamic guidance information. When the system is in normal operation, metro passenger's travel guidance mainly relies on the static guidance sign, and supplemented by a little dynamic guidance information. When an unexpected situation leads to the system operation abnormally, the passenger's travel plan adjustment need to rely on dynamic guidance information to complete. Therefore, the improvement level of metro system's dynamic guidance information have important influence to the network passenger flow's guidance and evacuation under abnormal conditions.

Dynamic guidance information is the guiding message to the passengers. It's effect both relate to release method and release environment, and relate to the passenger individual's visual sense, auditory sense and comprehension abilities. Compared with traditional static guidance signs, the dynamic guidance information that for the network passenger flow's guiding and evacuating is often semantic complex, diverse target and frequent change. So how to improve the dynamic information's release style and release method pertinently according to the passengers' characteristics and needs, so that achieve better guidance effect has become an urgent need solve problem during the process of enhance metro system's emergency treatment ability. 
This paper use questionnaire survey method, collect metro passengers evaluation data to common dynamic guidance information, based on the survey result, analyze the availability, clarity and comprehensibility characteristics of dynamic guidance information in various medium, find strengths and weaknesses of various types dynamic information, puts forward its application condition and improvement direction, and combine with the passengers' individual characteristics data, find out the passenger's attributes which influence the information's acceptability, so that provide help to make the pertinence passenger guidance scheme.

\section{Survey plan's design and implement}

The survey questionnaire of this survey include two parts, 17 questions in total. Part one is passenger's basic information, including:sex, age, education, occupation, income level, passenger use ticket's types, taken the subway's frequency and subway's familiarity degree. Part two is about dynamic information's acceptability, including: dynamic guidance information's dependent degree, dynamic guidance information's effect evaluation, the tendency of get dynamic guidance information's way, grade of each way's availability, problem of dynamic pictures, problem of broadcast, problem of roll titles, problem of display screen, deficiency of dynamic information.

The survey places select along Suzhou metro line 1 and 2, around the typical stations that have large passenger flows or have transfer function, such as Mudu, Suzhou Amusement Land, Xihuan Road, South Guangji Road, Leqiao Bridge, Donghuan Road, Xinghai Square, Xinghu Street which belongs to line 1; and Lumu, Pinglonglu East, Shi Lu, Shihu Donglu which belongs to line 2. From March to April 2016, carried out the questionnaire survey to the metro passengers at the above-mentioned locations. Use the way of give out questionnaires on-site and take back it immediately after answer finished, at last collected 231 effective questionnaires in total.

\section{Basic data's statistical analysis}

Statistic analysis of passenger's basic information

As the table 1 shows, the samples' distribution mainly conforms to the Suzhou urban area's total situation, and the survey objects generally contact and familiar with Suzhou subway, therefore, the survey results have good credibility. 
Table 1 Passenger's basic information statistical table

\begin{tabular}{|c|c|}
\hline Ques & Options and statistics (format: options (frequency/ percentage)) \\
\hline Sex & (1) $\operatorname{man}(133 / 57.6 \%)$; (2) woman(98/42.4\%) \\
\hline age & $\begin{array}{l}\text { (1) } 18 \text { year and below(4/1.7\%); (2) } 19-25 \text { year(47/20.3\%); (3) } 26-30 \text { year(46/ } \\
19.9 \%) ; \text { (4) } 31-35 \text { year(49/21.2\%); (5) } 36-40 \text { year(46/19.9\%); (6) } 41-45 \text { year(23 / } \\
10.0 \%) ; \text { ( } 456-50 \text { year(8/3.5\%); (8) } 51 \text { year and above( } 8 / 3.5 \%)\end{array}$ \\
\hline Educ & $\begin{array}{l}\text { (1)primary school (3/1.3\%); (2) junior high school (5/2.2\%); (3)senior high } \\
\text { school/technical school/technical secondary school (24/10.4\%); (4) junior } \\
\text { college/undergraduate course (167/72.3\%); (5)postgraduate (32/13.8\%) }\end{array}$ \\
\hline $\mathrm{Occ}$ & 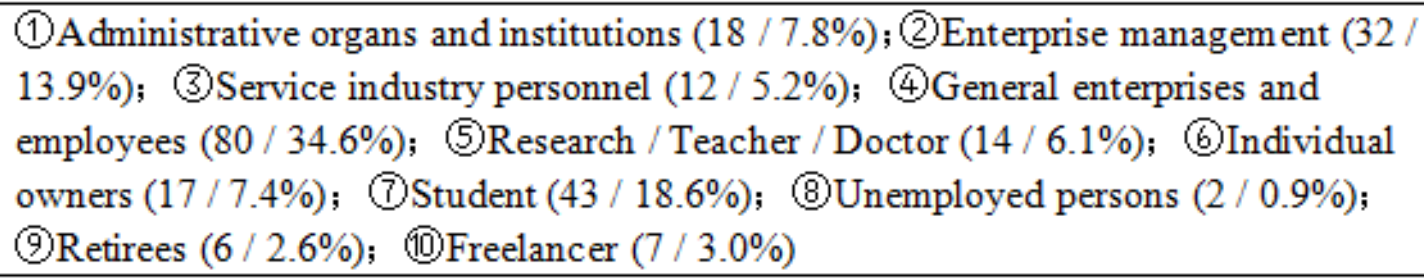 \\
\hline $\begin{array}{l}\text { Fam } \\
\text { mon } \\
\text { inco }\end{array}$ & $\begin{array}{l}\text { yuan and below (13/5.6\%); (2) } 1681-4000(44 / 19.1 \%) \text {; } \\
-8000(62 / 26.8 \%) ; \text { (4) } 8001-16000(67 / 29.0 \%) \text {; } \\
1-32000(37 / 16.0 \%) ; \quad(6) 32001 \text { and above(8/3.5\%); }\end{array}$ \\
\hline Tick & $\begin{array}{l}\text { (1)one-way ticket/counting card(64/27.7\%); (2) Ordinary transportation card (75/ } \\
32.5 \%) \text {; (3) Citizen card, social security card ( } 86 / 37.2 \%) \text {; (4) Students card, Seniors } \\
\text { card (6/2.6\%) }\end{array}$ \\
\hline $\begin{array}{l}\text { Taken } \\
\text { metro's } \\
\text { frequency }\end{array}$ & $\begin{array}{l}\text { (1) less than once a week }(52 / 22.5 \%) \text {; (2) } 1-4 \text { times a week( } 83 / 35.9 \%) \text {; } \\
\text { (3) } 5-9 \text { times a week(63/27.3\%); (4) } 10 \text { times a week and above(33/14.3\%) }\end{array}$ \\
\hline $\begin{array}{l}\text { Metro } \\
\text { familiarity } \\
\text { level }\end{array}$ & $\begin{array}{l}\text { (1) Completely unfamiliar, rarely taken (19/8.2\%); (2) Not too familiar, but generally } \\
\text { know how to find entrances and others ( } 46 / 19.9 \%) \text {; (3) Only familiar with the active } \\
\text { region's subway lines, not familiar with other areas ( } 90 / 39.0 \%) \text {; (4)Familiar with } \\
\text { most of Suzhou subway lines }(60 / 26.0 \%) \text {; (5)Familiar with the entire subway line in } \\
\text { Suzhou }(16 / 6.9 \%)\end{array}$ \\
\hline
\end{tabular}

Statistical analysis of dynamic information's acceptability

(1) passenger's dependence level on dynamic guidance information

As the figure 1 shows, passengers that consider dynamic information have more timely and clearer effect to guide passengers accounted for more than fifty percent, that consider the effect of dynamic information does not matter or generally only more than twenty percent, also have more than 10 percent passengers consider that it will be better if combined with static and dynamic. It indicates that most metro passengers hope that good dynamic guidance information will accompanied during the travel process to give well guide, that is, whether normal or abnormal conditions, dynamic guidance information can play a significant effect to enhance the passenger service level. 


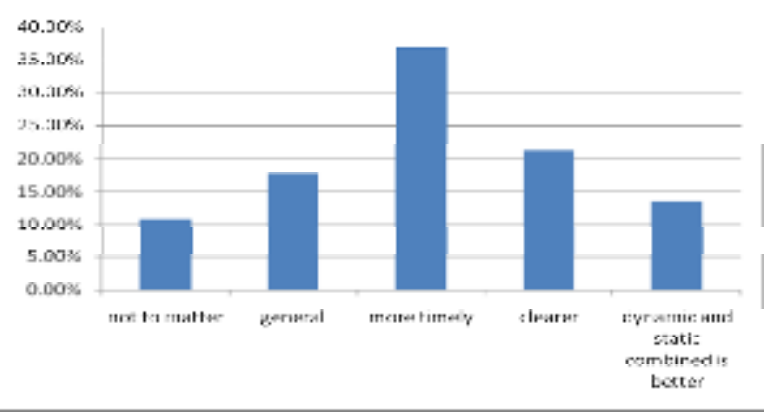

Figure 1 passenger's dependence level on dynamic guidance information

(2) passenger's effect evaluation to dynamic guidance information

As the figure 2 shows, more than seventy percent of the passengers consider that dynamic guidance information's content is sometimes difficult to understand and exist confusion; about ten percent of the passengers consider that dynamic guidance information is not clear, difficult to understand, and have poor effect; only less than ten percent of the passengers consider that the current dynamic guidance information is well. It suggested that passengers' effect evaluation to the current dynamic guidance information is not very high, hoping to improve the information's comprehensibility, so the subway should improve the dynamic guidance information's transmit mode, show style, and etc actively, so that make the meanings concisely and clearly.

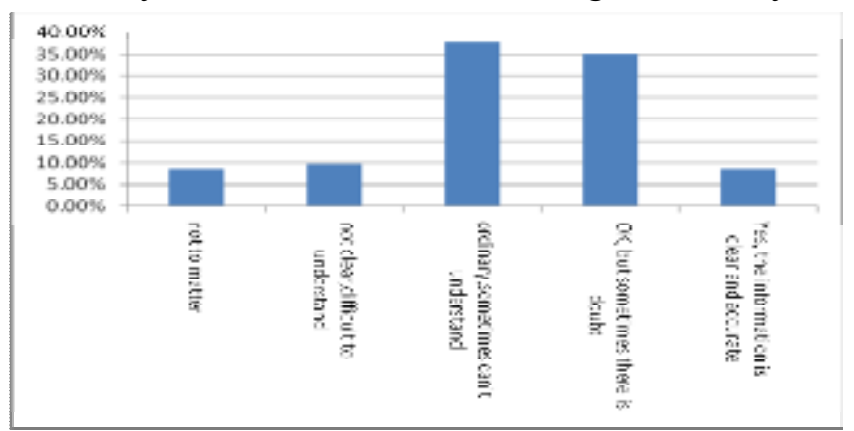

Figure 2 passenger's effect evaluation to dynamic guidance information

(3) the tendency of get dynamic guidance information's way

As figure 3 shows, at present, passengers mainly rely on dynamic pictures and display screen to get dynamic information, and employee prompt as auxiliary to complementary, three ways for more than seventy percent altogether, while the way of broadcast and roll titles' selection are only less than thirty percent. It indicates that passengers are more inclined to the mediums of picture, animation and video, which can express the complex information quickly and clearly, while mediums of voice and text are not conducive to passengers' accept because of its long express time, abstract content and other reasons.

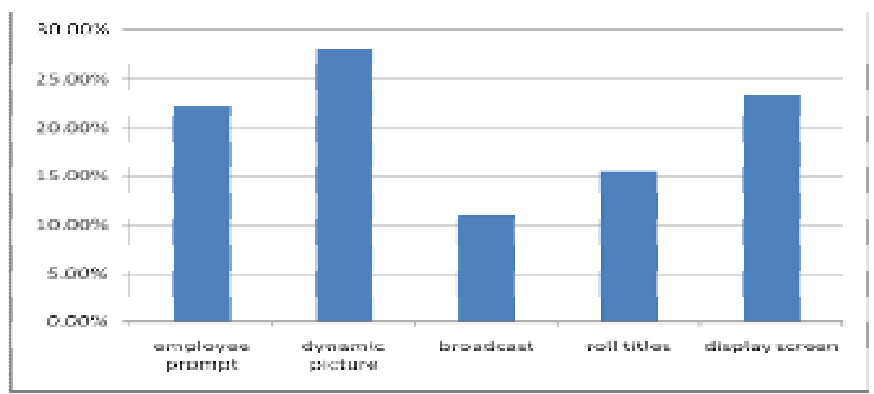

Figure 3 percentage of passengers' selection way to get dynamic guidance information 
(4) each way's availability

According to the difficulty degree of get the guidance information, from difficult to easy divide five grades, corresponding to 1 to 5 score, and let the metro passengers give scores to each way.

According to the Table 2's data, only the two ways of "employee prompt" and "dynamic picture" get the average score of more than 3, indicates that passengers are most likely and most easily through these two ways to get the required guidance information in the face of abnormal situations, which is mainly consistent with the passnegers' selection way results. At present, "display screen" is generally only installed at the side of platform, and the numbers are limited, far from full coverage various passenger flow lines, so although it has better effect, but its availability is poor, and can not undertake the main role of dynamic guide passengers under abnormal circumstance.

Table 2 the scores of each way to get guidance information's difficult level

\begin{tabular}{lcc}
\hline \multicolumn{1}{c}{ ways } & mean value & standard deviation \\
\hline employee prompt & 3.359 & 1.277 \\
dynamic picture & 3.273 & 1.328 \\
broadcast & 2.797 & 1.531 \\
roll titles & 2.606 & 1.437 \\
display screen & 2.978 & 1.356 \\
\hline
\end{tabular}

\section{Each way's insufficient and improvement}

(1) the dynamic picture way

As table 3 shows, the dynamic picture way's main problem is the content not clear and obscure, and then is the size problem and the boring style, only about ten percent passengers considered that there is no problem. Therefore, to improve dynamic picture's guide effect, works should be mainly around the picture's simple, clear and understandability, then supplemented by some flexible style. According to the above result, the dynamic picture is the main role of dynamic guiding passengers, so its arrangement range should cover the station's main passenger flow line, and its location should insure that all the passengers can easily view in the crowded situation.

Table 3 Dynamic picture way's survey results

\begin{tabular}{lcc}
\hline & frequency & percentage \\
\hline well, no problem & 41 & $10.9 \%$ \\
equipment is not advanced, display picture is not clear or incomplete & 88 & $23.4 \%$ \\
the picture size is not appropriate & 74 & $19.7 \%$ \\
content is too much or obscure & 110 & $29.2 \%$ \\
expreesion style is too boring & 63 & $16.8 \%$ \\
\hline
\end{tabular}

(2) the broadcast way

As table 4 shows, the broadcast's shortcomings is obviously, slow speed, too much content, less cycle index, and can not hear clearly are the problems passengers generally feedback, and these problems are difficult to solve only depend on broadcast itself. Therefore, the broadcast way can only be used as an auxiliary way to dynamic guiding passengers. It is more suitable for maintaining station's order and relieving passengers' emotion under the specific circumstances when passengers is not many and content is relative simple. 
Table 4 Broadcast way's survey results

\begin{tabular}{|c|c|c|}
\hline & frequency & percentage \\
\hline well, no problem & 37 & $9.8 \%$ \\
\hline speed is too slow, take too long to finish a sentence & 89 & $23.5 \%$ \\
\hline $\begin{array}{l}\text { the volume is not big enough, can not hear clearly when surrounding } \\
\text { noisy }\end{array}$ & 126 & $33.2 \%$ \\
\hline $\begin{array}{l}\text { have too much content and less cycle index, can not remember what to } \\
\text { say }\end{array}$ & 94 & $24.8 \%$ \\
\hline expression style is too boring or unclear & 33 & $8.7 \%$ \\
\hline \multirow{2}{*}{\multicolumn{3}{|c|}{$\begin{array}{l}\text { (3) roll titles way } \\
\text { As table } 5 \text { shows, scrolling speed is not appropriate, cycle time and waiting time too long are the } \\
\text { ain problems of this way, after that are the font size and definition problems. In view of same of } \\
\text { e problems are also difficult to completely resolved only depend on itself, this way should not be } \\
\text { e main way to dynamic guide passengers, and should be used in some stable information's loop } \\
\text { ayback. } \\
\text { Table } 5 \text { Roll titles way's survey results }\end{array}$}} \\
\hline & & \\
\hline & frequency & percentage \\
\hline well, no problem & 22 & $5.1 \%$ \\
\hline equipment is not advanced, displayed font is not clear or incomplete & 67 & $15.6 \%$ \\
\hline scrolling fonts' size are not appropriate & 64 & $14.9 \%$ \\
\hline scrolling speed is too fast or too slow & 117 & $27.2 \%$ \\
\hline content is ambiguous or uninteresting & 60 & $13.9 \%$ \\
\hline $\begin{array}{l}\text { the cycle of scrolling content is too long, waits too long for some } \\
\text { information }\end{array}$ & 100 & $23.3 \%$ \\
\hline
\end{tabular}

(4) display screen way

As table 6 shows, the display screen way's main problems, first are too many advertisements, too little facility number and too long cycle time, and then are the problems of poor picture quality, content complex and difficult to understand. Compared with other ways, this way has better flexibility, higher definition and better visual effect, the existing problems can be improved by itself, so it should play a more important role in the passengers' dynamic guidance. Besides platform, an appropriate number of display screens should be set above the passenger flow line inside train and subway hall, in peacetime arrange playback content according to operation needs, when fault occurs, it spread the network's real-time state information by full-screen pictures or animation style to all metro passengers, facilitate passengers to know the network status' real-time changes at any time, assist passengers' travel decisions. 
Table 6 Display screen way's survey results

\begin{tabular}{lcc}
\hline & frequency & percentage \\
\hline well, no problem & 33 & $7.9 \%$ \\
equipment is not advanced, the picture quality is poor or incomplete & 50 & $11.9 \%$ \\
the numble of screen is too little, size is too small, invisible and not clear & 76 & $18.2 \%$ \\
too many advertisements, affected view useful information & 101 & $24.2 \%$ \\
content is ambiguous or uninteresting & 40 & $9.6 \%$ \\
the cycle of scrolling content is too long, waits too long for some & 72 & $17.2 \%$ \\
information & & \\
information is too complex, not easy to understand immediately, do not & 46 & $11.0 \%$ \\
understand & & \\
\hline
\end{tabular}

\section{Impact analysis of passenger's attributes to guidance way's acceptability}

Take the dynamic guidance information's get way's tendency's survey result with passengers' basic attribute information's survey result together, carry on the bivariate correlation analysis, some of the results are shown in table 7. It indicate that only age, education level and taken subway's frequency will impact the acceptability of dynamic guidance way, but is not significance.

Table 7 Bivariate correlation analysis results

\begin{tabular}{|l|l|c|c|c|}
\hline \multicolumn{2}{|l|}{} & age & $\begin{array}{c}\text { Education } \\
\text { level }\end{array}$ & $\begin{array}{c}\text { taken subway's } \\
\text { frequency }\end{array}$ \\
\hline \multirow{2}{*}{$\begin{array}{l}\text { acceptance of dynamic } \\
\text { guidance way }\end{array}$} & $\begin{array}{l}\text { Pearson } \\
\text { correlation }\end{array}$ & $-0.137^{*}$ & $-0.138^{*}$ & $0.152^{*}$ \\
\cline { 2 - 5 } & $\begin{array}{l}\text { Significance } \\
\text { (two-tailed) }\end{array}$ & 0.038 & 0.036 & 0.021 \\
\cline { 2 - 5 } & $\mathbf{N}$ & 231 & 231 & 231 \\
\hline
\end{tabular}

Through the data statistics, it's can be find that elder people have relatively higher acceptance to the three ways of "employee prompt", "broadcast" and "roll titles", while have lower acceptance to "dynamic picture" and "display screen". Passnegers have higher education level prefer to "dynamic picture" and "display screen", while less educated are more likely to accept the other three ways. The acceptance of "employee prompt" decreased with the increase of taken subway's frequency, while the acceptance of "broadcast" and "roll titles" is conversely, the acceptance of "dynamic picture" and "display screen" does not change significantly with taken subway's frequency change.

\section{Conclusion and suggestions}

This paper from the investigation and analysis find that metro passengers have certain expectation on the dynamic guidance information's effect, hoping accompanied by perfect dynamic information during the travel process to improve the service experience. In the aspects of spread medium, passengers prefer to the fast and easy to understand ways such as picture or video, while sound and text ways' approbation degree are not very high. At the same time, passengers' evaluation to the existing dynamic guidance information system is not high, hoping to improve the information's understandability actively. "Dynamic picture" way should mainly make the contents simply, clearly and easy to understand; besides it, "display screen" way should increase the 
coverage and service more areas; "broadcast" and "roll titles" way should mainly responsible for the steady information's release, when passenger flow volume is less, these two ways can also properly undertake simple guidance information's release. When passenger flow volume is very high and situation is complex, in addition to the above-mentioned ways should cooperation, the "employee prompt" way should be applied in the station to satified certain passengers' guidance requirement. The analysis shows that passengers' age, education level and taken subway's frequency will affect its' dynamic guidance ways' acceptability, but not outstanding.

\section{Acknowledgements}

This paper is supported by National Natural Science Foundation young investigator grant program ( Grant No:51208328) .

\section{References}

[1] Lu Fang, Han Baoming, Cai Xiaochun (2012). “On the Behavior of Urban Rail Transit Regular Passengers". Urban Mass Transit, 02, 39-42.

[2] Liu Yongliang, Lijie (2012). "Design of the Subway Information Indicator System Based on Cognitive Psychology". Urban Rapid Rail Transit, 02, 39-42.

[3] J.M. Sullivan (2001). "Characteristics of Pedestrian Risk in Darkness". The University of Michigan Transportation Research Institute, 1-21.

[4] Steve M.V. Gwynne (1999). "An investigation of the aspects of occupant behavior required for evacuation modeling”. Journal of Applied Fire Science, 1, 18-59. 